\title{
A T-CONVERGENCE RESULT FOR VARIATIONAL INTEGRATORS OF LAGRANGIANS WITH QUADRATIC GROWTH
}

\author{
Francesco MagGi $^{1}$ And Massimiliano Morini $^{2}$
}

\begin{abstract}
Following the $\Gamma$-convergence approach introduced by Müller and Ortiz, the convergence of discrete dynamics for Lagrangians with quadratic behavior is established.
\end{abstract}

Mathematics Subject Classification. 37M15, 49J45.

Received October 7, 2003.

\section{INTRODUCTION}

In a recent paper Müller and Ortiz [4] have introduced the study of convergence properties of discrete dynamics and variational integrators using the tool of $\Gamma$-convergence. The theory of discrete dynamics is a formulation of Lagrangian mechanics in which time is considered as a discrete variable. This point of view leads to studying discrete trajectories, the variational integrators, generated by a discrete version of Hamilton's principle in which the classical integral action is replaced by a sum, called discrete action. The interest of this construction lies in the fact that, compared to the trajectories generated by more usual approximation schemes, variational integrators converge to classical solutions in a more stable way. We refer to Marsden and West [3] for a complete account and for more references on the topic.

In order to state and comment on the result by Müller and Ortiz [4], we introduce some definitions.

We say that a function $f: \mathbb{R}^{N} \times \mathbb{R}^{N} \rightarrow \mathbb{R}, f=f(s, \xi)$, is a Lagrangian with quadratic behavior if

$$
f \in C^{2}\left(\mathbb{R}^{N} \times \mathbb{R}^{N}\right) \text { with } \sup \left\{\left|D^{2} f(s, \xi)\right|:(s, \xi) \in \mathbb{R}^{N} \times \mathbb{R}^{N}\right\}<\infty,
$$

where $D^{2} f$ is the matrix of all the second order partial derivatives of $f$. Thus, for some $C>0$,

$$
|f(s, \xi)| \leq C\left(1+|s|^{2}+|\xi|^{2}\right), \quad \forall(s, \xi) \in \mathbb{R}^{N} \times \mathbb{R}^{N} .
$$

The action is the functional $F: H_{\mathrm{loc}}^{1}\left(\mathbb{R} ; \mathbb{R}^{N}\right) \times \mathcal{E} \rightarrow \mathbb{R}$, defined by

$$
F(u, A):=\int_{A} f(u, \dot{u}) \mathrm{d} t, \quad \forall(u, A) \in H_{\mathrm{loc}}^{1}\left(\mathbb{R} ; \mathbb{R}^{N}\right) \times \mathcal{E},
$$

\footnotetext{
Keywords and phrases. Discrete dynamics, variational integrators, Gamma-convergence.

1 Dipartimento di Matematica "U. Dini", Viale Morgagni 67/A 50134, Firenze, Italy.

2 Department of Mathematical Sciences, Carnegie Mellon University, Pittsburgh PA, 15213 USA;

e-mail: morini@asdf 4.math.cmu.edu
} 
where $\mathcal{E}$ is the family of all open bounded intervals of $\mathbb{R}$. A function $u$ is said to be stationary for the action if,

$$
\delta F(u, \varphi, A)=0, \quad \forall A \in \mathcal{E}, \forall \varphi \in C_{c}^{\infty}\left(A ; \mathbb{R}^{N}\right),
$$

where the first variation of $F(\cdot, A)$ on $u$ with respect to $\varphi$ is given by

$$
\delta F(u, \varphi, A):=\lim _{t \rightarrow 0} \frac{F(u+t \varphi, A)-F(u, A)}{t} .
$$

For every $h>0$ we fix the grid $\mathcal{T}_{h}=\{h i\}_{i \in \mathbb{Z}}$ of $\mathbb{R}$ of step $h$ and define the set $X_{h}$ of all $\mathcal{T}_{h}$-piecewise affine continuous functions with values in $\mathbb{R}^{N}$. A function $u_{h} \in X_{h}$ is said to be $h$-stationary for $F$ if

$$
\delta I\left(u_{h}, \varphi, A\right)=0, \quad \forall A \in \mathcal{E}, \forall \varphi \in X_{h}, \text { with } \operatorname{spt} \varphi \subset A .
$$

The main theorem in Müller-Ortiz [4] is the following:

Theorem 1. Assume that $f(s, \xi)=|\xi|^{2} / 2-V(s)$, where $V$ is of class $C^{2}$ and $\left|\nabla^{2} V\right|<C$ on $\mathbb{R}^{N}$. Let $\left\{u_{h}\right\}_{h}$ be a given sequence of $h$-stationary functions such that the Fourier transforms of the $u_{h}$ 's are in $L^{1}$ and form a compact sequence of Radon measures in the duality with $C_{b}\left(\mathbb{R} ; \mathbb{R}^{N}\right)$, the space of continuous and bounded functions on $\mathbb{R}$.

Then there exists $u \in W^{2, \infty}\left(\mathbb{R} ; \mathbb{R}^{N}\right)$ such that

a) $u_{h} \stackrel{*}{\rightarrow} u$ in $W^{1, \infty}\left(\mathbb{R} ; \mathbb{R}^{N}\right)$;

b) $u$ is stationary;

c) the Fourier transforms of the $u_{h}$ 's converge as measures in the flat norm to the Fourier transform of $u$.

The assumption made on the sequence of Fourier transforms implies that the sequence $\left\{u_{h}\right\}_{h}$ is uniformly bounded on $L^{\infty}\left(\mathbb{R} ; \mathbb{R}^{N}\right)$. Here we will deduce a similar property starting from the growth assumptions rather than supposing it.

The following theorem addresses these issues in a framework suitable to treat Lagrangians $f$ with quadratic behavior.

Theorem 2. Let $f$ be a Lagrangian with quadratic behavior, see (1), and let $f(s, \cdot)$ be uniformly convex, that is there exists $\nu>0$ such that

$$
\left\langle\frac{\partial^{2} f}{\partial \xi^{2}}(s, \xi) \eta, \eta\right\rangle \geq \nu|\eta|^{2}, \quad \forall s, \xi, \eta \in \mathbb{R}^{N} .
$$

Then there exists $h_{0}=h_{0}(C, \nu)$ such that

a) for every $\left(u_{0}, \xi_{0}\right) \in \mathbb{R}^{N} \times \mathbb{R}^{N}$ and $h<h_{0}$, there exists an $h$-stationary $u_{h}$ that is $h$-stationary, with $u_{h}(0)=u_{0},\left(u_{h}(h)-u_{h}(0)\right) / h=\xi_{0}$. Furthermore,

$$
\sup _{h<h_{0}}\left\|u_{h}\right\|_{W^{1, \infty}\left(A ; \mathbb{R}^{N}\right)} \leq K<\infty, \quad \forall A \in \mathcal{E},
$$

where $K$ is a constant depending only on $\left|u_{0}\right|,\left|\xi_{0}\right|, C, \nu, \mathcal{L}^{1}(A)$;

b) every sequence $\left\{u_{h}\right\}_{h}$, with $u_{h} h$-stationary and such that,

$$
\sup _{h} \max \left\{\left|u_{h}(0)\right|,\left|\left(u_{h}(h)-u_{h}(0)\right) / h\right|\right\}<\infty
$$

has a subsequence that converges weakly-* star in $W_{l o c}^{1, \infty}\left(\mathbb{R} ; \mathbb{R}^{N}\right)$ to a stationary $u$.

In Theorem 2 we are assuming that $f$ has the same growth in the $s$ and $\xi$ variables. A similar result cannot hold if $f$ has different growths with respect to $s$ and $\xi$. For example, let us consider $f(s, \xi)=\xi^{2} / 2+|s|^{2+\varepsilon} /(2+\varepsilon)$, $\varepsilon>0$. The Euler-Lagrange equation that characterizes the stationary functions is $\ddot{u}=u|u|^{\varepsilon}$, which has positive 
solutions for positive initial data. By conservation of the energy, for every solution $u$ with suitable initial data there exists a constant $C>0$ such that

$$
t=\int_{0}^{u(t)} \frac{\mathrm{d} u}{\sqrt{2 C+\frac{2}{2+\varepsilon} u^{2+\varepsilon}}}, \quad \forall t \in[0, \gamma]
$$

where $[0, \gamma]$ is the maximal interval of definition of $u$. Since $u\left(\gamma^{-}\right)=\infty$ we have

$$
\gamma=\int_{0}^{\infty} \frac{\mathrm{d} u}{\sqrt{2 C+\frac{2}{2+\varepsilon} u^{2+\varepsilon}}}<\infty
$$

The rest of the paper is devoted to the proof of Theorem 2. The first assertion in statement a) is proved in Section 2, while the second one, namely the bound (3), is proved in Section 3. In Section 4 we prove statement b) of Theorem 2 (here we follow [4]). In Section 5 we present a shorter proof of (3) that works for the prototype case where $f(s, \xi)=|\xi|^{2} / 2-V(s)$.

\section{EXISTENCE AND UNIQUENESS OF $h$-STATIONARY FUNCTIONS}

As a consequence of the assumption that $f$ is a Lagrangian with quadratic behavior, uniformly convex in the $\xi$ variable, there exist $C, \nu>0$ such that: for every $s, \xi \in \mathbb{R}^{N}$,

$$
\begin{aligned}
\nu|\xi|^{2}-C\left(1+|s|^{2}\right) \leq f(s, \xi) & \leq C\left(1+|s|^{2}+|\xi|^{2}\right), \\
\left|\frac{\partial f}{\partial s}(s, \xi)\right|+\left|\frac{\partial f}{\partial \xi}(s, \xi)\right| & \leq C(1+|s|+|\xi|), \\
\left|\frac{\partial^{2} f}{\partial s^{2}}(s, \xi)\right|+\left|\frac{\partial^{2} f}{\partial \xi^{2}}(s, \xi)\right|+\left|\frac{\partial^{2} f}{\partial s \partial \xi}(s, \xi)\right| & \leq C ;
\end{aligned}
$$

for every $s_{1}, s_{2}, \xi \in \mathbb{R}^{N}$,

$$
\left|\frac{\partial f}{\partial \xi}\left(s_{1}, \xi\right)-\frac{\partial f}{\partial \xi}\left(s_{2}, \xi\right)\right| \leq C\left|s_{1}-s_{2}\right|
$$

for every $s, \xi_{1}, \xi_{2} \in \mathbb{R}^{N}$,

$$
\left\langle\frac{\partial f}{\partial \xi}\left(s, \xi_{1}\right)-\frac{\partial f}{\partial \xi}\left(s, \xi_{2}\right), \xi_{1}-\xi_{2}\right\rangle \geq \nu\left|\xi_{1}-\xi_{2}\right|^{2} .
$$

We introduce the following notation for functions $u_{h} \in X_{h}$. We define for every $i \in \mathbb{Z}$

$$
u_{i}^{h}:=u_{h}(i h), \quad \xi_{i}^{h}:=\frac{u_{h}(i h+h)-u_{h}(i h)}{h}=\frac{u_{i+1}^{h}-u_{i}^{h}}{h} .
$$

Step 1. We claim that $u_{h} \in X_{h}$ is $h$-stationary if and only if, for every $i \in \mathbb{Z}$, it satisfies the following discrete Euler-Lagrange equation:

$$
\begin{array}{r}
0=\int_{0}^{1} t \frac{\partial f}{\partial s}\left(\left[u_{i}^{h}, u_{i+1}^{h}\right](t), \frac{u_{i+1}^{h}-u_{i}^{h}}{h}\right)+\frac{1}{h} \frac{\partial f}{\partial \xi}\left(\left[u_{i}^{h}, u_{i+1}^{h}\right](t), \frac{u_{i+1}^{h}-u_{i}^{h}}{h}\right) \mathrm{d} t \\
+\int_{0}^{1}(1-t) \frac{\partial f}{\partial s}\left(\left[u_{i+1}^{h}, u_{i+2}^{h}\right](t), \frac{u_{i+2}^{h}-u_{i+1}^{h}}{h}\right)-\frac{1}{h} \frac{\partial f}{\partial \xi}\left(\left[u_{i+1}^{h}, u_{i+2}^{h}\right](t), \frac{u_{i+2}^{h}-u_{i+1}^{h}}{h}\right) \mathrm{d} t,
\end{array}
$$


where $[a, b](t):=a+t(b-a)$ for every $a, b \in \mathbb{R}^{N}, t \in[0,1]$. Indeed, if $u$ is a piecewise affine function that takes the values $a, b, c \in \mathbb{R}^{N}$ at the points $i h,(i+1) h,(i+2) h$ respectively, then

$$
I(u,(i h,(i+2) h))=\int_{0}^{1} f\left([a, b](t), \frac{b-a}{h}\right) \mathrm{d} t+\int_{0}^{1} f\left([b, c](t), \frac{c-b}{h}\right) \mathrm{d} t=: L_{h}(a, b, c) .
$$

Taking a variation $\phi \in X_{h}$ which vanishes at $i h$ and $(i+2) h$ is equivalent to considering the function $\Phi(d):=$ $L_{h}(a, b+d, c)$ in that $u$ is stationary for this variation if and only if $\nabla \Phi(0)=0$, which gives exactly (10).

Step 2. For $a, b \in \mathbb{R}^{N}$ we define

$$
\begin{gathered}
\Psi_{a}^{h}(b):=\int_{0}^{1} \frac{\partial f}{\partial \xi}\left([a, b](t), \frac{b-a}{h}\right) \mathrm{d} t \\
M_{h}(a, b):=\int_{0}^{1} t h \frac{\partial f}{\partial s}\left([a, b](t), \frac{b-a}{h}\right)+\frac{\partial f}{\partial \xi}\left([a, b](t), \frac{b-a}{h}\right) \mathrm{d} t \\
N_{h}(a, b):=\int_{0}^{1} h(1-t) \frac{\partial f}{\partial s}\left([a, b](t), \frac{b-a}{h}\right) \mathrm{d} t .
\end{gathered}
$$

Then $(10)_{i}$ can be written as

$$
\Psi_{u_{i+1}^{h}}^{h}\left(u_{i+2}^{h}\right)=M_{h}\left(u_{i}^{h}, u_{i+1}^{h}\right)+N_{h}\left(u_{i+1}^{h}, u_{i+2}^{h}\right) .
$$

We claim that for every $a \in \mathbb{R}^{N}$, the function $\Psi_{a}^{h}: \mathbb{R}^{N} \rightarrow \mathbb{R}^{N}$ is a bijection with

$$
\operatorname{Lip}\left(\left(\Psi_{a}^{h}\right)^{-1}\right) \rightarrow 0 \quad \text { as } \quad h \rightarrow 0 .
$$

Indeed we have

$$
\begin{aligned}
\Psi_{a}^{h}(y)-\Psi_{a}^{h}(x)= & \int_{0}^{1} \frac{\partial f}{\partial \xi}\left([a, y](t), \frac{y-a}{h}\right)-\frac{\partial f}{\partial \xi}\left([a, x](t), \frac{x-a}{h}\right) \mathrm{d} t \\
= & \int_{0}^{1} d t \int_{0}^{1} \frac{d}{d r}\left\{\frac{\partial f}{\partial \xi}\left([a, x+r(y-x)](t), \frac{x+r(y-x)-a}{h}\right)\right\} \mathrm{d} r \\
= & \int_{0}^{1} d t \int_{0}^{1}\left\{\frac{\partial^{2} f}{\partial s \partial \xi}\left([a, x+r(y-x)](t), \frac{x+r(y-x)-a}{h}\right) \cdot(y-x)\right. \\
& \left.+\frac{\partial^{2} f}{\partial \xi^{2}}\left([a, x+r(y-x)](t), \frac{x+r(y-x)-a}{h}\right) \cdot \frac{(y-x)}{h}\right\} \mathrm{d} r .
\end{aligned}
$$

By the mean value theorem we can find $s=s(a, x, y), \xi=\xi(a, x, y, h)$, such that

$$
\Psi_{a}^{h}(y)-\Psi_{a}^{h}(x)=\frac{\partial^{2} f}{\partial s \partial \xi}(s, \xi) \cdot(y-x)+\frac{\partial^{2} f}{\partial \xi^{2}}(s, \xi) \cdot \frac{(y-x)}{h} .
$$

By (7) and (9) we have

$$
\left|\Psi_{a}^{h}(y)-\Psi_{a}^{h}(x)\right||y-x| \geq\left\langle\Psi_{a}^{h}(y)-\Psi_{a}^{h}(x), y-x\right\rangle \geq\left(\frac{\nu}{h}-C\right)|y-x|^{2} .
$$

If $h<\nu / C$ the surjectivity and injectivity of $\Psi_{a}^{h}$ follow from (12) at once, as well as

$$
\operatorname{Lip}\left(\left(\Psi_{a}^{h}\right)^{-1}\right) \leq\left(\frac{\nu}{h}-C\right)^{-1} \rightarrow 0 \quad \text { as } \quad h \rightarrow 0 .
$$


As a consequence for $h<\nu / C$ small enough we have that $(11)_{i}$ is equivalent to the fixed point problem $u_{i+2}^{h}=G^{h}\left[u_{i}^{h}, u_{i+1}^{h}\right]\left(u_{i+2}^{h}\right)$, where $G^{h}[a, b]: \mathbb{R}^{N} \rightarrow \mathbb{R}^{N}$ is defined by

$$
G^{h}[a, b](x):=\left(\Psi_{b}^{h}\right)^{-1}\left(M_{h}(a, b)+N_{h}(b, x)\right) .
$$

If $h<h_{0}=h_{0}(C, \nu)$ this map is a contraction. Indeed by $(7)$

$$
\left|\frac{\partial N_{h}}{\partial x}(b, x)\right| \leq h \int_{0}^{1}\left|\frac{\partial^{2} f}{\partial s^{2}}\left([b, x](t), \frac{x-b}{h}\right)+\frac{1}{h} \frac{\partial^{2} f}{\partial s \partial \xi}\left([b, x](t), \frac{x-b}{h}\right)\right| \mathrm{d} t \leq 2 C,
$$

and so

$$
\operatorname{Lip} G^{h}[a, b] \leq \operatorname{Lip}\left(\Psi_{b}^{h}\right)^{-1} \operatorname{Lip} N_{h}(b, \cdot) \leq 2 C\left(\frac{\nu}{h}-C\right)^{-1} .
$$

By the Banach-Caccioppoli theorem for every $a, b \in \mathbb{R}^{N}$ there exists a unique $x \in \mathbb{R}^{N}$ such that $x=G^{h}[a, b](x)$.

Step 3. Let $h<h_{0}$ and fix $u_{0}, \xi_{0} \in \mathbb{R}^{N}$. Then there exists a unique $h$-stationary point $u_{h} \in X_{h}$ such that $u_{0}^{h}=u_{0}, \xi_{0}^{h}=\xi_{0}$. Indeed, under these initial conditions we have $u_{1}^{h}=u_{0}^{h}+h \xi_{0}^{h}$, and then, in view of step 2, we can uniquely determine $u_{2}^{h}$ from $u_{0}^{h}, u_{1}^{h}$ by solving $x=G^{h}\left[u_{0}^{h}, u_{1}^{h}\right](x)$. By induction we can define $u_{h}$ on $[0, \infty)$ in such a way that $(10)_{i \in \mathbb{N}}$ are satisfied. Similarly, we may define $u_{h}$ on $(-\infty, 0]$ so that the equations $(10)_{i \in \mathbb{Z}}$ hold.

\section{Proof of LOCAL Boundedness of $h$-STATionary SEQUENCES}

Step 1. For each $h<h_{0}$ we consider the $h$-stationary point $u_{h}$ with $u_{0}^{h}=u_{0}$ and $\xi_{0}^{h}=\xi_{0}$. Let us write the $i$ th discrete Euler-Lagrange equation as $A_{i}^{h}=B_{i}^{h}$ where

$$
\begin{aligned}
& A_{i}^{h}:=\int_{0}^{1} \frac{\partial f}{\partial \xi}\left(\left[u_{i+1}^{h}, u_{i+2}^{h}\right](t), \xi_{i+1}^{h}\right)-\frac{\partial f}{\partial \xi}\left(\left[u_{i}^{h}, u_{i+1}^{h}\right](t), \xi_{i}^{h}\right) \mathrm{d} t, \\
& B_{i}^{h}:=h \int_{0}^{1} t \frac{\partial f}{\partial s}\left(\left[u_{i}^{h}, u_{i+1}^{h}\right](t), \xi_{i}^{h}\right)+(1-t) \frac{\partial f}{\partial s}\left(\left[u_{i+1}^{h}, u_{i+2}^{h}\right](t), \xi_{i+1}^{h}\right) \mathrm{d} t .
\end{aligned}
$$

Since $|[a, b](t)-[b, c](t)| \leq|a-b|+|b-c|$, we have that

$$
\begin{aligned}
\left|A_{i}^{h}\right| \geq & \left|\int_{0}^{1} \frac{\partial f}{\partial \xi}\left(\left[u_{i+1}^{h}, u_{i+2}^{h}\right](t), \xi_{i+1}^{h}\right)-\frac{\partial f}{\partial \xi}\left(\left[u_{i+1}^{h}, u_{i+2}^{h}\right](t), \xi_{i}^{h}\right) \mathrm{d} t\right| \\
& -\left|\int_{0}^{1} \frac{\partial f}{\partial \xi}\left(\left[u_{i+1}^{h}, u_{i+2}^{h}\right](t), \xi_{i}^{h}\right)-\frac{\partial f}{\partial \xi}\left(\left[u_{i}^{h}, u_{i+1}^{h}\right](t), \xi_{i}^{h}\right) \mathrm{d} t\right| \\
& \geq \nu\left|\xi_{i+1}^{h}-\xi_{i}^{h}\right|-C h\left(\left|\xi_{i}^{h}\right|+\left|\xi_{i+1}^{h}\right|\right),
\end{aligned}
$$

and by $(6)$,

$$
\left|B_{i}^{h}\right| \leq C h\left(1+\left|u_{i}^{h}\right|+h\left|\xi_{i}^{h}\right|+\left|\xi_{i}^{h}\right|\right)+C h\left(1+\left|u_{i+1}^{h}\right|+h\left|\xi_{i+1}^{h}\right|+\left|\xi_{i+1}^{h}\right|\right) .
$$

Since $\left|u_{i}^{h}\right| \leq\left|u_{0}\right|+h \sum_{j=0}^{i-1}\left|\xi_{j}^{h}\right|$ and $A_{i}^{h}=B_{i}^{h}$, a straightforward computation leads from (13) and (14) to

$$
\left|\xi_{i+1}^{h}-\xi_{i}^{h}\right| \leq \frac{2 C h}{\nu}\left(1+\left|u_{0}\right|+h \sum_{k=0}^{i}\left|\xi_{k}^{h}\right|+\left|\xi_{i}^{h}\right|+\left|\xi_{i+1}^{h}\right|+h\left|\xi_{i+1}^{h}\right|\right) .
$$


Let us define $\sigma_{i}^{h}:=\max \left\{\left|\xi_{j}^{h}\right|: 0 \leq j \leq i\right\}$. We have

$$
\begin{aligned}
\sum_{i=0}^{j}\left|\xi_{i+1}^{h}-\xi_{i}^{h}\right| & \leq \frac{2 C h}{\nu}\left\{(j+1)\left(1+\left|u_{0}\right|\right)+h \sum_{i=0}^{j} \sum_{k=0}^{i}\left|\xi_{k}^{h}\right|+\sum_{i=0}^{j}\left(\left|\xi_{i}^{h}\right|+\left|\xi_{i+1}^{h}\right|\right)+h \sum_{i=0}^{j}\left|\xi_{i+1}^{h}\right|\right\} \\
& \leq \frac{2 C(j+1) h}{\nu}\left\{\left(1+\left|u_{0}\right|\right)+h(j+1) \sigma_{j}^{h}+2 \sigma_{j}^{h}+h \sigma_{j}^{h}\right\}+\frac{2 C}{\nu}\left(h+h^{2}\right)\left|\xi_{j+1}^{h}\right| .
\end{aligned}
$$

Since $\left|\xi_{j+1}^{h}\right| \leq\left|\xi_{0}\right|+\sum_{i=0}^{j}\left|\xi_{i+1}^{h}-\xi_{i}^{h}\right|$, we obtain

$$
\left\{1-\frac{2 C}{\nu}\left(h+h^{2}\right)\right\}\left|\xi_{j+1}^{h}\right| \leq\left|\xi_{0}\right|+\frac{2 C(j+1) h}{\nu}\left\{\left(1+\left|u_{0}\right|\right)+(h(j+1)+h+2) \sigma_{j}^{h}\right\} .
$$

Choose $h_{0}=h_{0}(C, \nu)$ such that for $h<h_{0}$ we have $\left(1-(2 C / \nu)\left(h+h^{2}\right)\right) \geq 1 / 2$ and for $R_{0}:=2 h_{0}$ we have $\left(4\left(R_{0}+h_{0}\right) C / \nu\right)\left(R_{0}+2 h_{0}+2\right) \leq 1 / 2$. For every $j \in\left\{0, \ldots,\left[R_{0} / h\right]\right\}$ we have $(j+1) h \leq R_{0}+h$, and thus

$$
\left|\xi_{j+1}^{h}\right| \leq 2\left|\xi_{0}\right|+\frac{4\left(R_{0}+h\right) C}{\nu}\left(1+\left|u_{0}\right|\right)+\frac{4\left(R_{0}+h\right) C}{\nu}\left(R_{0}+2 h+2\right) \sigma_{j}^{h} \leq L+\sigma_{j}^{h} / 2
$$

if we define

$$
L=L\left(u_{0}, \xi_{0}, C, \nu\right):=2\left|\xi_{0}\right|+\left(4\left(R_{0}+h_{0}\right) C / \nu\right)\left(1+\left|u_{0}\right|\right)
$$

By (15) we conclude that for every $h<h_{0}$ and for every $j \in\left\{0, \ldots, N_{h}\right\}$, where $N_{h}:=\left[R_{0} / h\right]$, we have

$$
\sigma_{j+1}^{h} \leq \max \left\{\sigma_{j}^{h}, L+\sigma_{j}^{h} / 2\right\}
$$

From (16) it follows that

$$
\begin{aligned}
\sigma_{j+1}^{h} & \leq \max \left\{\sigma_{j}^{h}, L+\sigma_{j}^{h} / 2\right\} \leq \max \left\{\sigma_{j-1}^{h}, L+\sigma_{j-1}^{h} / 2, L+\sigma_{j}^{h} / 2\right\} \\
& \leq \max \left\{\sigma_{j-1}^{h}, L+\sigma_{j}^{h} / 2\right\} \leq \ldots \leq \max \left\{\sigma_{0}, L+\sigma_{j}^{h} / 2\right\},
\end{aligned}
$$

where $\sigma_{0}^{h}=\left|\xi_{0}^{h}\right|=\left|\xi_{0}\right|=: \sigma_{0}$. Below we write $N$ instead of $N_{h}$ for simplicity of notations and we iterate (17) to get,

$$
\begin{aligned}
\sigma_{N+1}^{h} & \leq \max \left\{\sigma_{0}, L+2^{-1} \sigma_{N}^{h}\right\} \\
& \leq \max \left\{\sigma_{0}, L+2^{-1} \max \left\{\sigma_{0}, L+2^{-1} \sigma_{N-1}^{h}\right\}\right\} \\
& =\max \left\{\sigma_{0}, L+2^{-1} \sigma_{0}, L\left(1+2^{-1}\right)+2^{-2} \sigma_{N-1}^{h}\right\} \\
& \leq \max \left\{\sigma_{0}+2 L, L\left(1+2^{-1}\right)+2^{-2} \sigma_{N-1}^{h}\right\} \\
& \leq \max \left\{\sigma_{0}+2 L, L\left(1+2^{-1}\right)+2^{-2} \max \left\{\sigma_{0}, L+2^{-1} \sigma_{N-2}^{h}\right\}\right\} \\
& \leq \max \left\{\sigma_{0}+2 L, L\left(1+2^{-1}\right)+2^{-2}\left(L+2^{-1} \sigma_{N-2}^{h}\right)\right\} \\
& =\max \left\{\sigma_{0}+2 L, L\left(1+2^{-1}+2^{-2}\right)+2^{-3} \sigma_{N-2}^{h}\right\} \\
& \leq \cdots \\
& \leq \max \left\{\sigma_{0}+2 L, L \sum_{j=0}^{N} 2^{-j}+2^{-N+1} \sigma_{0}\right\} \leq \sigma_{0}+2 L .
\end{aligned}
$$

Thus, for $h<h_{0}$, we have

$$
\sup _{\left[0, R_{0}\right]}\left|\dot{u}_{h}\right| \leq\left|\sigma_{N_{h}+1}^{h}\right| \leq \sigma_{0}+2 L
$$


which implies

$$
\sup _{h<h_{0}}\left\|u_{h}\right\|_{W^{1, \infty}\left(\left[0, R_{0}\right]\right)} \leq K\left(\max \left\{\left|u_{0}\right|,\left|\xi_{0}\right|\right\}, C, \nu\right),
$$

with $K$ increasing in its first variable. To complete the proof of (3) it remains to show that for every $M>0$,

$$
\sup _{h<h_{0}}\left\|u_{h}\right\|_{W^{1, \infty}([0, M])} \leq K^{*}\left(\max \left\{\left|u_{0}\right|,\left|\xi_{0}\right|\right\}, C, \nu, M\right) .
$$

Indeed we can cover $[0, M]$ with a finite union of intervals of the form $I_{i}:=t_{i}+\left[0, R_{0}\right], t_{i}<t_{i+1}, t_{1}=0$ such that $I_{i} \cap I_{i+1}$ has at least length $h_{0}$. By (18),

$$
\sup _{h<h_{0}}\left\|u_{h}\right\|_{W^{1, \infty}\left(I_{1}\right)} \leq K\left(\max \left\{\left|u_{0}\right|,\left|\xi_{0}\right|\right\}, C, \nu\right) .
$$

This is the case $j=1$ of the following family of inequalities indexed by $j$, that we are going to prove by induction; precisely, we claim that,

$$
\sup _{h<h_{0}}\left\|u_{h}\right\|_{W^{1, \infty}\left(I_{j}\right)} \leq K_{j}\left(\max \left\{\left|u_{0}\right|,\left|\xi_{0}\right|\right\}, C, \nu\right)<\infty .
$$

Let us assume this holds for $j-1$. Since the problem is autonomous we can apply (18) to get

$$
\sup _{h<h_{0}}\left\|u_{h}\right\|_{W^{1, \infty}\left(I_{j}\right)} \leq K\left(\max \left\{\left|u_{h}\left(\inf I_{j}\right)\right|,\left|\dot{u}_{h}\left(\left(\inf I_{j}\right)\right)\right|\right\}, C, \nu\right) .
$$

On the other hand, since inf $I_{j} \in I_{j-1}$, by the inductive hypothesis we have

$$
\begin{aligned}
K\left(\max \left\{\left|u_{h}\left(\inf I_{j}\right)\right|,\left|\dot{u}_{h}\left(\left(\inf I_{j}\right)\right)\right|\right\}, C, \nu\right) \leq & K\left(K_{j-1}\left(\max \left\{\left|u_{0}\right|,\left|\xi_{0}\right|\right\}, C, \nu\right), C, \nu\right) \\
=: & K_{j}\left(\max \left\{\left|u_{0}\right|,\left|\xi_{0}\right|\right\}, C, \nu\right),
\end{aligned}
$$

and the assertion is proved. Since with a finite number of steps we can cover $[0, M],(19)$ is established.

\section{Proof of Theorem 2, part B}

The proofs of this section follow closely the ideas of Müller and Ortiz [4].

Step 1. We first prove the following: there exists a constant $l>0$ depending only on $C$ and $\nu$ such that, if $Y$ is a linear subspace of $H_{\text {loc }}^{1}\left(\mathbb{R} ; \mathbb{R}^{N}\right)$ and $u \in Y$ is stationary with respect to variations in $Y$, i.e.,

$$
\delta F(u, \varphi, A)=0, \quad \forall A \in \mathcal{E}, \forall \varphi \in Y, \text { with } \operatorname{spt} \varphi \subset A,
$$

then $u$ minimizes $F(\cdot, A)$ among all functions $v \in Y$ with $u_{\mid \partial A}=v_{\mid \partial A}$, for every $A \in \mathcal{E}$ such that $\mathcal{L}^{1}(A)<l$. In fact we will need to apply this property only for $Y=X_{h}$.

Let $u$ be such that $(20)$ holds, $A=(a, b) \in \mathcal{E}$ and let $v \in Y$ such that $\varphi:=v-u$ is zero on $\partial A$. Let us consider the function

$$
g(r):=\int_{a}^{b} f(u+r \varphi, \dot{u}+r \dot{\varphi}) \mathrm{d} t
$$

By (20) we have that $g^{\prime}(0)=0$ and by Taylor's Formula we obtain

$$
\begin{aligned}
\int_{a}^{b} f(u+\varphi, \dot{u}+\dot{\varphi}) \mathrm{d} t-\int_{a}^{b} f(u, \dot{u}) \mathrm{d} t & =\int_{0}^{1} g^{\prime \prime}(r)(1-r) \mathrm{d} r \\
& =\int_{0}^{1}(1-r) \int_{a}^{b} Q[r, t](\varphi(t), \dot{\varphi}(t)) \mathrm{d} t \mathrm{~d} r
\end{aligned}
$$


where $Q[r, t]: \mathbb{R}^{n} \times \mathbb{R}^{n} \rightarrow \mathbb{R}$ is the quadratic form defined by

$$
\begin{aligned}
Q[r, t](\rho, \tau):= & \left\langle\frac{\partial^{2} f}{\partial s^{2}}(u(t)+r \varphi(t), \dot{u}(t)+r \dot{\varphi}(t)) \rho, \rho\right\rangle+2\left\langle\frac{\partial^{2} f}{\partial s \partial \xi}(u(t)+r \varphi(t), \dot{u}(t)+r \dot{\varphi}(t)) \rho, \tau\right\rangle \\
& +\left\langle\frac{\partial^{2} f}{\partial \xi^{2}}(u(t)+r \varphi(t), \dot{u}(t)+r \dot{\varphi}(t)) \tau, \tau\right\rangle
\end{aligned}
$$

By (7) and (2) it is easy to see that

$$
Q[s, t](\rho, \tau) \geq \nu|\tau|^{2}-C|\rho|^{2}-2 C|\rho||\tau| \geq \frac{\nu}{2}|\tau|^{2}-\left(C+2 C C^{\prime}\right)|\rho|^{2}
$$

where $C^{\prime}$ is a constant depending only on $\nu$ and $C$. Therefore by (21) and by Poincaré's Inequality we get

$$
\begin{aligned}
\int_{a}^{b} f(u+\varphi, \dot{u}+\dot{\varphi}) \mathrm{d} t-\int_{a}^{b} f(u, \dot{u}) \mathrm{d} t & \geq \int_{0}^{1}(1-r) \int_{a}^{b}\left(\frac{c}{2}|\dot{\varphi}(t)|^{2}-\left(C+2 C C^{\prime}\right)|\varphi(t)|^{2}\right) \mathrm{d} t \mathrm{~d} r \\
& \geq \int_{0}^{1}(1-r)\left(\frac{c}{2} \frac{\pi^{2}}{(b-a)^{2}}-C-2 C C^{\prime}\right) \int_{a}^{b}|\varphi(t)|^{2} \mathrm{~d} t \mathrm{~d} r \\
& >0
\end{aligned}
$$

where the last inequality holds provided that $(b-a)^{2}<\left(c \pi^{2}\right) /\left(2 C+4 C C^{\prime}\right)=: l$.

Step 2. Let us consider a sequence $\left\{u_{h}\right\}_{h}$ with each $u_{h} h$-stationary and such that (4) hold. By (3) and by Ascoli-Arzelá compactness criterion we infer that, up to extracting a subsequence, $u_{h} \stackrel{*}{\rightarrow} u$ in $W_{\text {loc }}^{1, \infty}\left(\mathbb{R} ; \mathbb{R}^{N}\right)$, where $u \in W_{\text {loc }}^{1, \infty}\left(\mathbb{R} ; \mathbb{R}^{N}\right)$. We want to prove that $u$ is $h$-stationary for the action. Clearly it suffices to show that

$$
F(u, A) \leq F(v, A), \quad \forall v \in H_{\mathrm{loc}}^{1}\left(\mathbb{R} ; \mathbb{R}^{N}\right), \text { with } \operatorname{spt}(v-u) \subset A,
$$

for every $A \in \mathcal{E}$ with $\mathcal{L}^{1}(A)<l$, where $l$ is defined as in step 1 . It is in this part of the proof that a $\Gamma$-convergence argument is used.

Let us fix such an interval $A$ and a function $v$. By (5) and by (2) we can apply De Giorgi-Ioffe lower semicontinuity theorem [1], [2]) to see that

$$
F(u, A) \leq \liminf _{k \rightarrow \infty} F\left(w_{k}, A\right), \quad \forall\left\{w_{k}\right\}_{k} \subset H^{1}\left(A ; \mathbb{R}^{N}\right), w_{k} \rightarrow u \mathrm{w}-H^{1}\left(A ; \mathbb{R}^{N}\right) .
$$

In particular

$$
F(u, A) \leq \liminf _{h \rightarrow 0} F\left(u_{h}, A\right) .
$$

On the other hand, by Lemma 4.2.b in [4] there exists $\left\{v_{h}\right\}_{h} \subset X_{h}$ with $v_{h}=v$ on $\partial A$ such that $v_{h} \rightarrow v$ strongly in $H^{1}\left(A ; \mathbb{R}^{N}\right.$ ) (in particular the convergence is strong in $L^{\infty}$ ). By (5) and the strong convergence of the $v_{h}$ 's we have that

$$
F(v, A)=\lim _{h \rightarrow 0} F\left(v_{h}, A\right)
$$

In order to prove $F(u, A) \leq F(v, A)$ and to conclude the proof we need to compare the behavior of $\left\{F\left(u_{h}, A\right)\right\}_{h}$ and $\left\{F\left(v_{h}, A\right)\right\}_{h}$. Clearly, if $v_{h}=u_{h}$ on $\partial A$, we conclude by a direct application of the result in step 1 for $Y=X_{h}$ combined with (22) and (23). If this is not the case we can argue as follows. Let us consider $\left\{A_{h}\right\}_{h} \subset \mathcal{E}$ such that $A_{h}=\left(a_{h}, b_{h}\right) \subset A=(a, b), A_{h}$ is compatible with $\mathcal{T}_{h}$ and $a_{h} \rightarrow a, b_{h} \rightarrow b$. Since $u\left(a_{h}\right)-v\left(a_{h}\right) \rightarrow 0$ and $u\left(b_{h}\right)-v\left(b_{h}\right) \rightarrow 0$, there exists a sequence of affine functions $\left\{p_{h}\right\}_{h}$ such that

$$
v_{h}+p_{h}=u_{h} \quad \text { on } \quad \partial A_{h}, \quad p_{h} \rightarrow 0 \quad \text { uniformly. }
$$


Let $w_{h}:=v_{h}+p_{h}$ on $A_{h}$ and $w_{h}=u_{h}$ on $A \backslash A_{h}$. By the minimality of $u_{h}$ on $A_{h}$ proved in step 1 we now get

$$
F\left(u_{h}, A_{h}\right) \leq F\left(w_{h}, A_{h}\right) .
$$

We claim that

$$
F(u, A) \leq \liminf _{h \rightarrow 0} F\left(u_{h}, A_{h}\right), \quad \lim _{h \rightarrow 0} F\left(w_{h}, A_{h}\right)=F(v, A),
$$

clearly this will conclude the proof. The first inequality comes from (22) and the fact that $F\left(u_{h}, A \backslash A_{h}\right) \rightarrow 0$ by (5) and (3). The second inequality holds since $\left|F\left(w_{h}, A_{h}\right)-F\left(v_{h}, A_{h}\right)\right|=\left|F\left(v_{h}+p_{h}, A_{h}\right)-F\left(v_{h}, A_{h}\right)\right| \rightarrow 0$ as $h \rightarrow 0$. This is an easy consequence of the properties of $f$ and of the uniform convergence of $\left\{p_{h}\right\}_{h}$ to zero.

\section{A Short PROOF OF (3) IN THE MOdEL CASE}

Here we give a short proof of (3) in the model case $f(s, \xi)=|\xi|^{2} / 2-V(s)$, where $V$ is as in Theorem 1. Let us consider a $h$-stationary $u_{h}$ such that $u_{0}^{h}=u_{0}$ and $\xi_{0}^{h}=\xi_{0}$. It is easy to see that the discrete Euler-Lagrange equations can now be written as

$$
\xi_{i+1}^{h}=h \int_{0}^{1} t \nabla V\left(\left[u_{i}^{h}, u_{i+1}^{h}\right](t)\right)+(1-t) \nabla V\left(\left[u_{i+1}^{h}, u_{i+2}^{h}\right](t)\right) \mathrm{d} t+\xi_{i}^{h} .
$$

Since the second derivatives of $V$ are uniformly bounded on $\mathbb{R}^{N}$ we have $|\nabla V|(s) \leq C(1+|s|)$, and so we get

$$
\left|\xi_{i+1}^{h}\right| \leq C h\left(1+\left|\left[u_{i}^{h}, u_{i+1}^{h}\right]\right|+\left|\left[u_{i+1}^{h}, u_{i+2}^{h}\right]\right|\right)+\left|\xi_{i}^{h}\right| .
$$

As $\left|u_{i+1}^{h}\right| \leq\left|u_{i}^{h}\right|+h\left|\xi_{i}^{h}\right|$ it follows that

$$
\begin{aligned}
\left|\xi_{i+1}^{h}\right| & \leq C h\left(1+\left|u_{i}^{h}\right|+h\left|\xi_{i}^{h}\right|+\left|u_{i}^{h}\right|+h\left|\xi_{i}^{h}\right|+h\left|\xi_{i+1}^{h}\right|\right)+\left|\xi_{i}^{h}\right| \\
& \leq 2 C h\left(1+\left|u_{i}^{h}\right|\right)+\left(1+2 C h^{2}\right)\left|\xi_{i}^{h}\right|+C h^{2}\left|\xi_{i+1}^{h}\right|,
\end{aligned}
$$

so that

$$
\left|\xi_{i+1}^{h}\right| \leq A_{h}\left|\xi_{i}^{h}\right|+B_{h}\left(1+\left|u_{i}^{h}\right|\right), \quad A_{h}:=\frac{1+2 C h^{2}}{1-C h^{2}}, \quad B_{h}:=\frac{2 C h}{1-C h^{2}} .
$$

Since $\left|u_{i}^{h}\right| \leq\left|u_{0}\right|+h \sum_{j=0}^{i-1}\left|\xi_{j}^{h}\right|$, if we define $\sigma_{i}^{h}:=\max \left\{\left|\xi_{j}^{h}\right|: 1 \leq j \leq i\right\}$, we get,

$$
\left|\xi_{i+1}^{h}\right| \leq\left(A_{h}+i h B_{h}\right) \sigma_{i}^{h}+B_{h}\left(1+\left|u_{0}\right|\right) .
$$

Let $R>0$. For $1 \leq i \leq[R / h]$ by the previous equation we have

$$
\left|\xi_{i+1}^{h}\right| \leq\left(A_{h}+R B_{h}\right) \sigma_{i}^{h}+B_{h}\left(1+\left|u_{0}\right|\right) .
$$

Since $A_{h}>1$, setting $D_{h}:=\left(A_{h}+R B_{h}\right)>1$ we conclude

$$
\begin{aligned}
\sigma_{i+1}^{h} & \leq D_{h} \sigma_{i}^{h}+B_{h}\left(1+\left|u_{0}\right|\right) \leq D_{h}^{2} \sigma_{i-1}^{h}+B_{h}\left(1+\left|u_{0}\right|\right)\left(1+D_{h}\right) \\
& \leq \ldots \leq D_{h}^{i+1} \sigma_{0}+B_{h}\left(1+\left|u_{0}\right|\right) \sum_{j=0}^{i} D_{h}^{j} \leq D_{h}^{i+1}\left(\sigma_{0}+i B_{h}\left(1+\left|u_{0}\right|\right)\right) .
\end{aligned}
$$

Then

Since

$$
\left\|\dot{u}_{h}\right\|_{L^{\infty}(0, R)} \leq \sigma_{[R / h]+1}^{h} \leq D_{h}^{[R / h]+2}\left(\sigma_{0}+([R / h]+1) B_{h}\left(1+\left|u_{0}\right|\right)\right) .
$$

$$
D_{h}^{[R / h]+2}=\exp \left(([R / h]+2) \log \left(A_{h}+R B_{h}\right)\right)=\exp (([R / h]+2)(2 C R h+o(h))),
$$


and $\left\{([R / h]+1) B_{h}\right\}_{h}$ is bounded, we have that the right hand side of $(25)$ is bounded for $h$ small by a constant depending on $C, R,\left|u_{0}\right|$ and $\left|\xi_{0}\right|$. This proves (3) in this special case.

Acknowledgements. We thank Irene Fonseca for proposing us the problem. This work was supported by the Center for Nonlinear analysis under NSF Grant DMS-9803791. The first author was supported by the Ph.D. program of the University of Florence and by MIUR Cofin 2003-2004 through the research project "Equazioni e sistemi differenziali nonlineari degeneri: esistenza e regolaritá".

\section{REFERENCES}

[1] E. De Giorgi, Teoremi di semicontinuitá nel Calcolo delle Variazioni. Istituto Nazionale di Alta Matematica (1968-1969).

[2] A.D. Ioffe, On lower semicontinuity of integral functionals. I. SIAM J. Control Optim. 15 (1977) $521-538$.

[3] E.J. Marsden and M. West, Discrete Mechanics and variational integrators. Acta Numerica 10 (2001) 357-514.

[4] S. Müller and M. Ortiz, On the $\Gamma$-convergence of discrete dynamics and variational integrators. J. Nonlinear Sci. 14 (2004) $279-296$. 